\title{
Accessible Publications Checklist
}

\section{Introduction}

The Accessible Publications Checklist serves a guide to creating scholarly publications with accessibility and universal design in mind. Universality benefits everyone, not just people with disabilities. This checklist overlaps with other best practices in usability, interaction design, web and graphic design, search engine optimization, and user studies. More broadly, the publishing of accessible research corresponds to equity, diversity and inclusion within the context of scholarly communication as it promotes inclusive information design practices for equitable access to information.

\section{Considerations}

The checklist is not meant to be an exhaustive list of requirements, rather a curated set of design tips for content authors or editors to use during the publication production phase of research (one phase of the scholarly communication process). Although the checklist can be applied to various word processing software used to create publications, the open source software Libre Office Writer is used as the basis for examples. Similarly, although the checklist can be applied to various types of publications (books, OER, articles, dissertations, etc), the journal article is the basis for examples used in the checklist.

\section{Table of Contents}

Introduction

Considerations. 1

Create Text that is Organized, Readable and Understandable ...........................................................

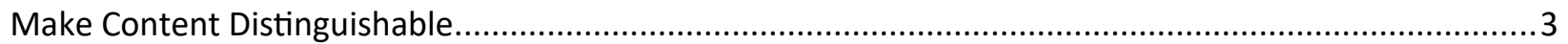

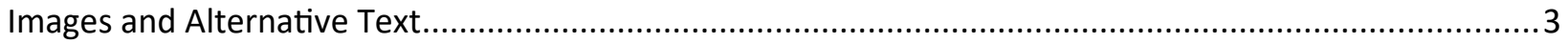

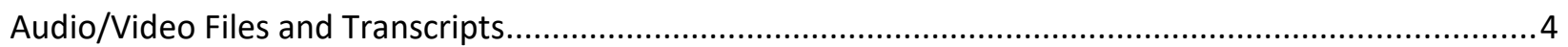

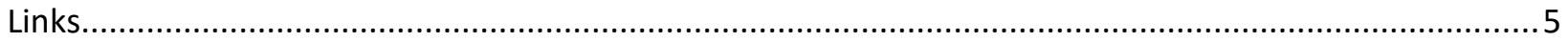

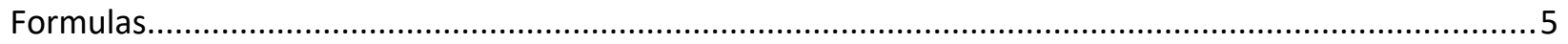

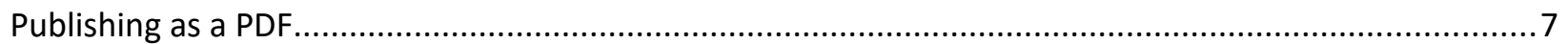

Examples of Accessible Publications and Accessibility Checkers.......................................................

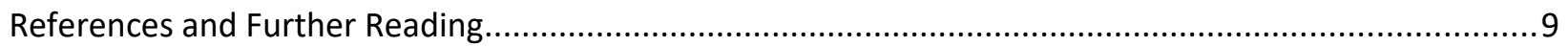




\section{Create Text that is Organized, Readable and Understandable.}

- Use semantic headings (and with HMTL for electronic publishing) and a hierarchy document structure, enabling assistive devices and readers to navigate the content with ease. The following best practices are applicable to document creation in word processors and spreadsheets:

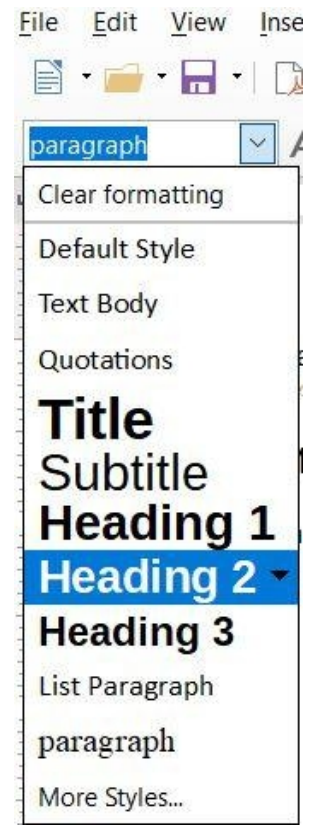

Figure 1: Libre Office Headings Level Tags
- HTML helps create accessible content as it adapts to different browsers and devices but it also provides a logical flow to main concepts presented in the content. Headings $(\mathrm{H} 1, \mathrm{H} 2, \mathrm{H} 3)$ are available for use to structure content in word processing software such as Microsoft Office, Open Office, Libre Office, Google Docs, and others.

- It is important to the use the heading level tags instead of making the text appear like a heading by using bold text or larger font. See Figure 1.

- Ensure the default font sizes are not too small and can be expanded or zoomed in on websites or PDFs. Recommended sizes include 12 point font for body text and 9 to 10 point for footnotes. Headings and subheadings will be larger than body text, depending on the default settings of your word processor, although these can be manually adjusted to your preferred size.

- If using lists, tables, or input fields, make sure these are marked up properly. For example, for lists, actually use the software's bullet list or numbers list functions rather than placing images of bullets or dashes to a create the visual appearance of a list. Tables should include properly defined column headers and rows.

- Use a variety of access points for navigation (whether a book or journal), such as lists of tables or figures, table of contents, index or search capabilities. 


\section{Make Content Distinguishable}

Adhering to all of these may not be possible or needed, depending on the publication type.

Examples actions that can help make content distinguishable include:

- Limit the use of color as the only way of conveying information

- Use sufficient contrast between foreground and background colors

- Making sure text is not lost when users change between viewpoints (making text larger and smaller)

- Images and text of images are resize-able by the user

- Audio can be paused, stopped and volume adjusted by user (if applicable)

- Avoid using content that flashes or warn users in advance if flashing will occur

\section{Images and Alternative Text}

- Use alternative text (commonly referred to as 'alt tag' or 'alt text') and have this copyedited with the manuscript. The alt text is a short description of the visual resource, often 120 characters or less. A long description can also be supplied if there is a specific need for longer description, such as the structural elements within a table.

- When creating descriptions of images, the alt text must convey the content and its functionality. Rather than describing what an image looks like, state what it is and what it does. Keep it short, just one or two sentences is adequate. Unless it is a complex image, such as a highly detailed map, more context in the description may be necessary to convey is purpose. For examples on complex image descriptions, see PCC (2020) Complex Image Accessibility.

- Examples of images that need alt text, include icons, buttons, graphics, charts, diagrams, illustrations, audio/video files, labels for form controls, input, or user interface components.

- When alt text is used, the information is available to people who are blind and are visible to search engines and people who turn off images which may affect people concerned with costs or low bandwidth (W3C, "Accessibility"). 
- Use high-quality alt text or descriptions for the front cover images of a journal issue and article (if applicable). This cover alt text description can be placed on the journal's copyright page and is the responsibility of the journal editorial team (not authors).

- Accessible information design practices can vary by media format. For guidance on how to write descriptions for an image, see Alternative Text by WebAIM or $\underline{\text { Image Description }}$ Guidelines by Diagram Center. Keep in mind images that are merely decorative (not part of the comprehension of the document) do not need alt text. Therefore, if the image's purpose is decorative rather than informative, then avoid using unnecessary alt text descriptions.

- When describing the image, make sure color is not the only distinguishing element. Use high-contrast colors to make shading differences more apparent so they are still visible in gray-scale and use text labels in the image to help convey information. If interested in learning how to select contrast colors, see WebAIM's Contrast Checker to test your color options.

- For deeper knowledge on accessible images, see the various guidelines developed by WGBH, including Accessible Digital Media Guidelines, Effective Practices for Description of Science Content with Digital Talking Books (includes examples of descriptions for a bar chart, line graph, Venn Diagram, scatter plot, table, pie chart, flow chart, illustrations and math equations), and Describing Images for Enhanced Assessments. All of these are available at the WGBH website.

- Cadet, a free caption and description editing tool by the National Center for Accessible Media at WGBH is available online.

\section{Audio/Video Files and Transcripts}

- Provide transcripts for audio files. People who have hearing difficulties are able to access the audio content, as well as search engines or other technologies without the capabilities of hearing (W3C, "Accessibility").

- Transcripts should include the speaker's name (if known), content, and relevant descriptions. 
- Provide captions for video files, where the audio is synchronized with the video. If captioning is not possible, provide a transcript of the audio in the video and descriptions of any contextual visuals seen in the video.

- If embedding a video not produced by the author (for example, using a video created by a third-party, such as Youtube), then be careful not to violate any potential copyright issue. Check with the copyright holder to obtain permission before using the video and creating a transcript or captions.

- Captioning can be done for a fee by vendors or can be done on your own. Free resources for creating captions include Youtube and Amara. For advice on creating captions, see Accessible Digital Media Guidelines for Multimedia by WGBH or Captions, Transcripts and Audio Descriptions by WebAIM.

\section{Links}

- Make sure links are accessible. For examples and guidelines, see the Links section of the Accessibility Toolkit by Coolidge et. al (2018). This includes describing the purpose of the link within the text so there is meaningful context. Avoid using unhelpful text such as "click here."

- Example of helpful link description:

More information on the W3C Web Accessibility Initiative is available online.

\section{Formulas}

- $\quad$ Single-line equations should be expressed in unicode fonts. Multi-line equations are often captured as images with long descriptions of alt text and although some software can assist in writing multi-line equations for insertion into journals or books, they are not always readable by ebook devices.

- LaTex (open source typesetting program) can be used to write equations and is typically transmitted to Braille devices but MathML is more widely accepted by 
browser and device types. An alternative to MathML is simply using an image of the equation with an alt text description or an audio file describing the equation. To learn more about this, see the Formulas section of the Accessibility Toolkit by Coolidge et. al (2018) or the Equations: MathML, Images, and LaTex from Accessibility and Usability by Penn State University.

- It is recommended that any formulas used in journal articles be written electronically by the authors (not the library or publishers). The author can use their preferred equation software or use Libre Office's Writer with the following steps. See Figure 2 for a visual aid, which is described with the following steps:

- First, select Insert from the menu bar, then Object and Formula.

- Second, the formula can be pasted into the bottom text input area.

- Third, if the equation is not yet written, use the features of the system (including the drop down menu)and select among operators to add to the text input area.

- Fourth, as you make selections and edit the formula in the input area, the resulting formula will become visible in the text of the word processor. When the formula is complete, click in the text document to return to editing.

- These steps can also be used by the journal publisher if the author gives the electronic formula to the journal who will just paste it into the text input area as described in the steps above when working in Libre Office. 


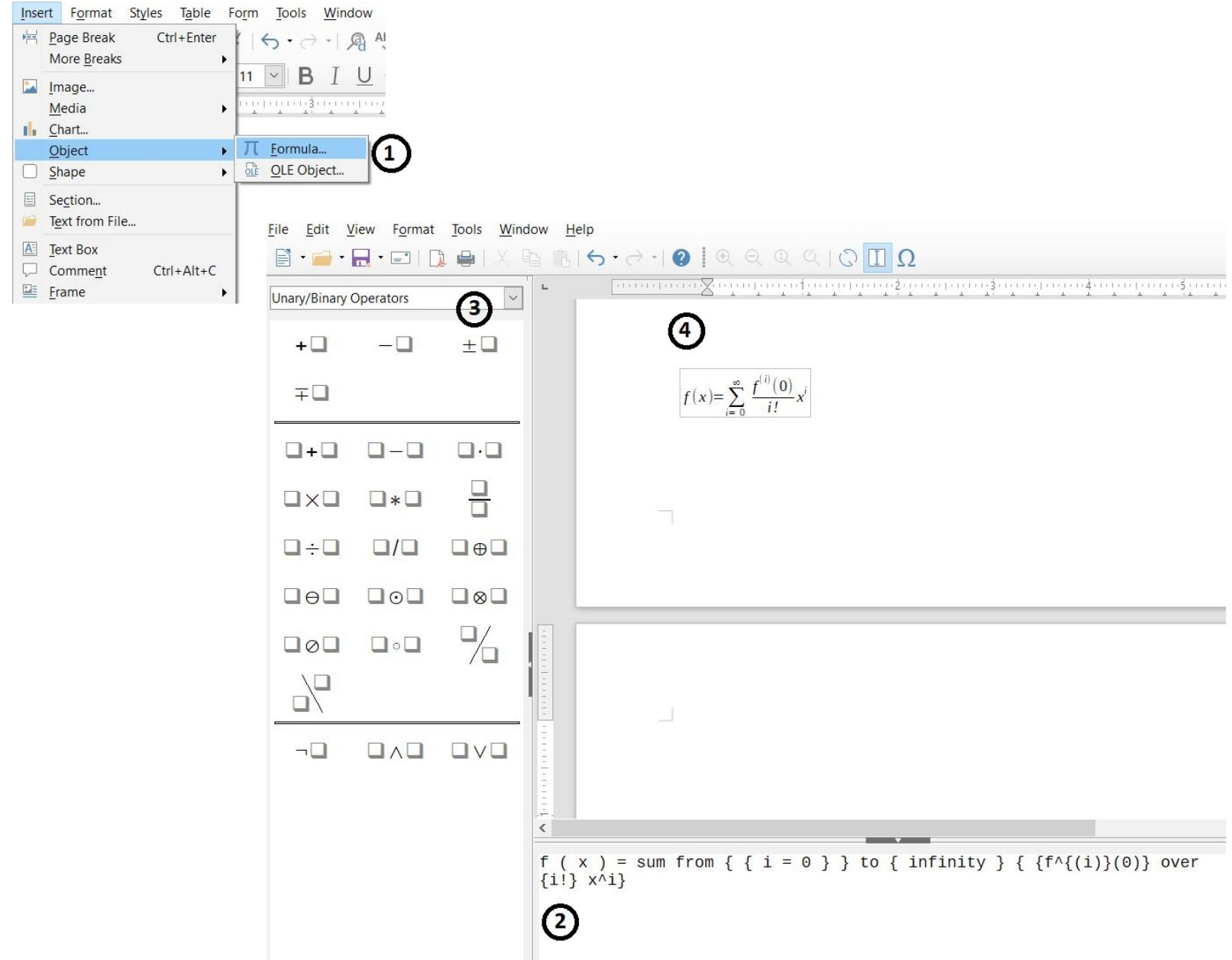

Figure 2: Insert Equation Formula in Libre Office

\section{Publishing as a PDF}

- If a document is scanned and exported as a PDF (rather than created in word processing software), the document may simply be an image of text. This is not ideal, as the result will lack optical character recognition (OCR). OCR makes the document machine readable (search engines need OCR to read the text and users need OCR to search the document electronically). You must perform OCR on the document during the scanning process.

- If exporting a document to PDF from other formats, such as Microsoft Office or Libre Office, verify the source document has semantic structures in place as stated earlier in 
this checklist and add the necessary metadata describing the document, such as author, title, and other pertinent publishing information.

- To add this metadata in Libre Office, from File, select Properties. Then from the open dialog box, select the Description tab to enter in the title, author and other relevant information. To add more detailed metadata (optional), select the Custom Properties tab and Add Property (ie: date, publisher, or something else you choose). Click OK when finished. See Figure 3 for a visual aid.

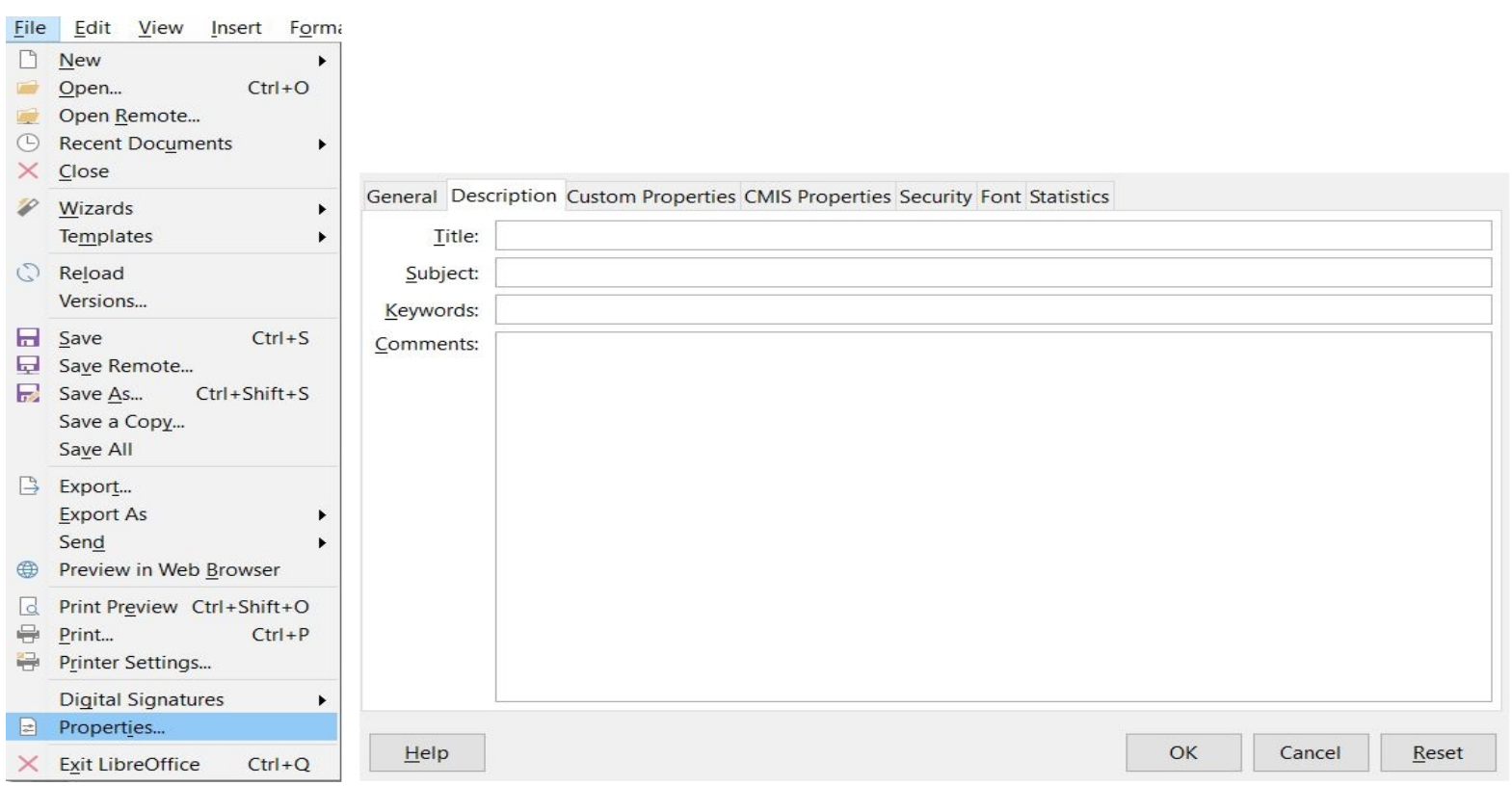

Figure 3: Libre Office Metadata Description

- Resources for creating accessible PDFs include PDF Accessibility by WebAIM, Create and Verify PDF Accessibility by Adobe Acrobat, and Create Accessible PDFs by Microsoft. Instructions at these sites advise users to make sure the PDF's document structure tags and create bookmarks using headings are selected as options during the export process.

- Publishing a journal issue or article as HTML can be done in a text editor, such as Dreamweaver, Atom.io, Visual Studio Code, or Notepad++. Alternatively, the manuscript or journal issue can be exported as HTML from the word processor being used, such as Microsoft Office or Libre Office. The resulting HTML file can then be placed in the journal 
repository or website hosting the content. Use of HTML for publishing is not the focus of this checklist, however, you can check if your HTML is accessible by using the WAVE Web Accessibility Evaluation Tool online.

\section{Examples of Accessible Publications and Accessibility Checkers}

- For an example of the anatomy of an accessible page, see page 2 of the Web Accessibility Guidelines by Portland Community College.

- For assistance in creating accessible digital products (such as documents, websites,

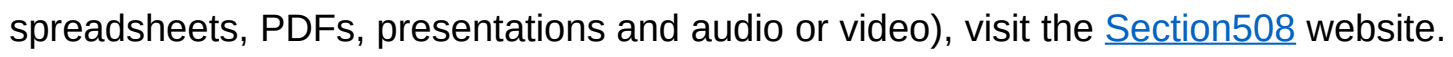

- Certain software has built-in accessibility checkers, including Microsoft Office and Adobe Acrobat. Browsers can also be used to confirm screen readers can read PDFs created with accessibility in mind. Once you create an accessible PDF, test it out by opening it with a browser, such as Mozilla. Right click in the document (in the browser) and click "read aloud" to see if your document is machine readable. 


\section{References and Further Reading}

\section{References}

Adobe Acrobat. (2019). Create and verify PDF accessibility.

https://helpx.adobe.com/acrobat/using/create-verify-pdf-accessibility.html

Coolidge, A., Doner, S., Robertson, T., \& Gray, J. (2018). Accessibility Toolkit - 2nd Edition.

Victoria, B.C.: BCcampus. Retrieved from https://opentextbc.ca/accessibilitytoolkit/

Diagram Center. (2019). Image description guidelines. http://diagramcenter.org/table-ofcontents-2.html

Libre Office Writer. https://www.libreoffice.org/

Microsoft Office. (n.d.). Create accessible PDFs. https://support.office.com/en-us/article/Createaccessible-PDFs-064625E0-56EA-4E16-AD71-3AA33BB4B7ED

Penn State University. (n.d.). Equations: MathML, images, and LaTex.

http://accessibility.psu.edu/equations

Portland Community College (2020). Complex Image Accessibility.

https://www.pcc.edu/instructional-support/accessibility/images/

Portland Community College. (2015). Web Accessibility Handbook, $2^{\text {nd }}$ Edition.

https://www.pcc.edu/instructional-support/accessibility/handbook/

W3C (2018). Accessibility. https://www.w3.org/standards/webdesign/accessibility.html

WebAIM. (2020). Alternative text. https://webaim.org/techniques/alttext/

WebAIM. (2020). Captions, transcripts and audio descriptions

https://webaim.org/techniques/captions/

WebAIM. (2020). Contrast checker. https://webaim.org/resources/contrastchecker/

WebAIM. (2020). PDF accessibility. https://webaim.org/techniques/acrobat/converting

WebAIM. (n.d.). WAVE Web Accessibility Evaluation Tool. https://wave.webaim.org/ 
WGBH. (2020). Accessible digital media guidelines.

https://www.wgbh.org/foundation/ncam/guidelines/accessible-digital-media-guidelinesintroduction

\section{Further Reading}

Use the Accessible Publications Checklist as a starting point and keep in mind that it is best to work with colleagues who are web designers, publishers, and/or accessibility specialists when possible. Standards applicable to accessibility, websites, and universal design include:

U.S. Federal government's Section 508 rules, https://www.section508.gov

Title II of the Americans with Disabilities Act (ADA) and Section 504 of the Rehabilitation Act of 1973 (as amended in 1998)

Web Content Accessibility Guidelines (WCAG) https://www.w3.org/WAl/standards-guidelines/wcag

Parts of this checklist were adapted from various sources including author experience and works created by colleagues and educational institutions, including:

BC Campus. OER Accessibility Toolkit. (last edited 2018).

https://wiki.ubc.ca/Documentation:Open_UBC/Guide/OER_Accessibility_Toolkit

Miller, A. (2019). Digital Project Preservation Plan: A Guide for Preserving Digital

Humanities/Scholarship Projects. https://jewlscholar.mtsu.edu/handle/mtsu/5761

Portland Community College. (2015). Web Accessibility Handbook, $2^{\text {nd }}$ Edition.

https://www.pcc.edu/instructional-support/accessibility/handbook/ 